\title{
Determine the Environment and the Efficacy of Genuine Traditional Chinese Medicine
}

\author{
M.Bai, S.Tian, M.S.Miao*, J.J.Jiao, Y.Li, W.Y.Xin \\ Henan University of Traditional Chinese Medicine, Henan, 450008, china
}

\begin{abstract}
The genuine traditional Chinese medicine as a synonym for quality medicine, it has a long history in China, it has important applications in the aspects of disease prevention and treatment of traditional Chinese medicine. This paper from the growth conditions of genuine traditional Chinese medicine, such as geographical location, for students status, etc to study its growing conditions. Study different growth conditions and growth state influence on the efficacy of medicinal, clarify the understanding of genuine traditional Chinese medicine, and the relation of its growth conditions and efficacy, clarify the characteristics of the original ecological planting, pointed out that the efficacy of genuine traditional Chinese medicine and their growing conditions has closely related, we should depth study the relationship of the efficacy of genuine traditional Chinese medicine and their growing conditions, increase the research of the original ecological planting, ensure the quality of medicines from the source, promote the sustainable use of genuine traditional Chinese medicine, and promote the development traditional Chinese Medicine Enterprise.
\end{abstract}

KEYWORD: The genuine traditional Chinese medicine; Environmental; Efficacy

\section{INTRODUCTION}

Traditional Chinese medicine in the Chinese nation has a thousands years history, it is a treasure of the Chinese nation. In recent years, due to the degree of attention of Chinese medicine continues to improve, the genuine traditional Chinese medicine are increasingly attracted much attention, multidisciplinary theory and technology is gradually applied to the study of the genuine traditional Chinese medicine, making the scientific connotation of the genuine traditional Chinese medicine become more clear. growth conditions as an important factor for the formation of genuine traditional Chinese medicine, it has played an important role in the formation of quality and the clinical treatment. Original ecological planting is simulated growing conditions of the genuine traditional Chinese medicine, making a artificial planting of genuine traditional Chinese medicine, so as to ensure the effectiveness and sustainability of the use of clinical medicine. This paper discusses the growth conditions impact on the genuine traditional Chinese medicine and the influence of different growth conditions on the medicinal efficacy.

\section{THE GENUINE TRADITIONAL CHINESE MEDICINE}

The genuine traditional Chinese medicine as an unique quality standards for the evaluation of traditional Chinese medicine unique quality standards, its idea deeply rooted in traditional Chinese medicine theory, it is the ancient physicians of clinical validation and summary. The concept first seen in "Shen Nong's Herbal Classic", there has "the time of harvesting and manufacturing, raw and cooked, the land, authenticity, old and new, and each method,"[1].The so-called genuine traditional Chinese medicine, also known as authentic ingredients, it is a synonymous with high and pure quality herbs, it refers from specific areas, long history of production, has a fine planting and processing techniques, high quality, a significant effect, with a geographical features herbs, it is the result of the effect on history and clinical examination was evaluated as good quality herbs[2].

Records relating to genuine traditional Chinese medicine as early as the Qin Dynasty had already started to appear, as in "Fifty-two Diseases" had mentioned "pansy" growing in swamp side. After the Qin unification, along with social and economic development, herbs exchanges are also increasing, most of the writings of this period are recorded on 
medicinal origin, such as "Mingyi bielu", documented the Rehmannia "grows in Xianyang Kawasawa Yellow Earth were better," etc, it has begun to take the basic characteristics of genuine medicine at this time. Southern and Northern Dynasties, the famous herbal book "Annotations of Materia Medica", it has records a lot situation about genuine traditional Chinese medicine , and explained the substance of genuine medicine is the quality, efficacy superior to other place, then the actual content of genuine traditional Chinese medicine has been formed. Tang and Song dynasties, China's first herbal book issued by the official "New Revised Materia Medica," the preface had pointed out, "the Animal and Plant Health ... from its native, the same quality and efficiency differences", which shows that people on herbs knowledge provenance, and the number and the understanding of genuine traditional Chinese medicine has also been further developed, to the Ming and Qing Dynasties, in Tang Xianzu "The Peony Pavilion", clearly put forward the term "genuine traditional Chinese medicine"[3].In modern society, as people demand genuine traditional Chinese medicine increases, the original ecological planting herbs proposed, simulated wild environment, ensure the quality of medicine, meet the needs of clinical and sustainable development of genuine traditional medicine.

In addition, the genuine traditional Chinese medicine is not static, it is also with the changing social and natural conditions, the "genuine" origin is also undergoing constant change. Such as ancient herbal records, Anemarrhena mainly in Hebei, Shanxi, Henan area, but now mainly produced in Hebei Xiling [4], indicating that with the change of natural and geographical conditions, the genuine origin has changed.

\section{THE GENUINE TRADITIONAL CHINESE MEDICINE AND GROWTH CONDITIONS}

The natural herbs of distribution and production are inseparable from certain natural conditions, in the order of Sun Simiao's "valuable prescriptions for emergency" said: "the ancient medicine...... Medication will be in accordance with the land, so the treatment of ten to nine", it fully illustrate the effect of the origin of medicinal herbs to the quality. Different regions, different growth conditions, suitable for different plant growth, so only good quality medicines in certain areas, it can become genuine traditional Chinese medicine. Specific ecological environmental conditions is an important external factors for the form of genuine traditional Chinese medicine[5], these specific growth environment conditions include light, temperature, moisture, air, topography, soils, topography and other factors, these factors are closely related with the growth and development, efficacy, quality, not only affects the quality of medicines, but also affect the synthesis of active ingredients of Chinese herbal medicines[6]. Such as when the mint grown in sandy soil, which has a high oil content, Mandala grown in alkaline soils, the high alkaloid content[7]. Light is an important condition for photosynthesis of plants, water is an important condition for the medicinal plants and medicinal animals to survive and development, soil is the basis for the growth of Chinese herbal medicines, the landform and physiognomy although not a direct influence, but can restrict light, temperature, moisture and other natural factors, for the medicinal plants or medicinal animal's survival still plays a decisive role. Therefore, the original ecological planting of Chinese herbal medicines also require herbs to grow their particular, in response to the growth environment, it can form a unique medicinal qualities. If it violate the laws of nature grow, it will be counterproductive, although produced a lot of herbs, but lost the unique qualities, it can not meet the needs of clinical. In addition, some medications has no toxicity, but with the transition of times and the change of growth conditions, the drug itself in order to survive and produce some active substances, and these active substances become effective ingredient of the drug. Such as Gelsemium, it is not toxic, but with the change of the natural environment, resulting in toxicity, and these toxic components also become the material basis of its efficacy. There are some drugs, the historical record is not toxic, but with the development of modern science and technology, and then found its toxicity, Such as Aster, there is no ancient records of its toxicity, but modern research proves that excessive use, it will produce significant toxicity symptoms, but the reason of causes toxicity remains to be further study [8].

The genuine traditional Chinese medicine grown in certain ecological environment, its potency will surely be affected by the environment. Herbs such as growth in the water more than cold, with the role of diuresis. In addition, the season of harvesting herbs are also closely related to the quality, modern research shows that the alkaloids in Ephedra in autumn was the highest, rutin content in Sophora japonica is higher in flower bud period. Nature as an ecological balance system, rely on the existence and the relationship between any plants and other plants and to maintain the balance of nature. Similarly, Chinese herbal medicine is only maintained homeostasis between other plants, animals, in order to maintain their own growth, to ensure the efficacy. 


\section{THE EFFICACY AND GROWTH CONDITIONS}

The ancients warned "Where the administration who must choose the appropriate land, with the drug, with the evidence ...... if not choice, treat wasting its power, the end will not be living (Song" Ben Cao Yan Yi "), fully explains the different medicinal origin, its efficacy has also vastly different. Under normal circumstances, plants accumulate organic matter, it is often the active ingredients of play their efficacy and treatment. It can be seen, different growth conditions for the formation of the quality of medicine and its clinical efficacy has negligible impact[9], such as climate, topography, land form soil and other factors. Medicinal quality is closely related to the local climate, soil, topography and other ecological factors[10],such as Alisma and other aquatic herbs, only grow in moist and goo soil, it can ensure their active ingredients, to ensure its efficacy. Studies have found that the quality of soil is closely related to the quality of herbs, such as artemisinin, its content has a significant positive relationship with the calcium content of the soil. Efficacy is the ultimate standard that identification and evaluation of the quality of the genuine traditional Chinese medicine. In improving clinical efficacy, the genuine traditional Chinese medicine is the most effective weapon of TCM.As Arnebia euchroma, comfrey and Inner Mongolia comfrey comfrey root can be used as medicine, Chinese medicine believes Arnebia clinical applications for the gifted, antibacterial efficacy also showed Arnebia euchroma> Inner comfrey> comfrey [11]. Analysis of the impact of climate ecological factors on American ginseng active ingredient content, the results indicate that temperature and sunlight are the major climatic factors affecting American ginseng total saponin content[12]. Modern research has also shown that microbial has a closely related with the active ingredients of herbs[13], efficacy of plant has a related with the microorganisms of secondary metabolites contained in its type and number, it is affected by the type and content of secondary metabolites in plants to affect its medicinal properties. Such as Resina Draconis is a plant antitoxin formed after fungal invasion or injured, and healthy Dracaena were very low, and now with the fungus-induced method to produce blood, it can solve the problem of shortage of raw materials.

In addition, the symbiotic endophytes and plants, can cause changes in plant metabolism, generally believed that endophytes can quickly and highly specific and selective induction of plant express the specific genes, thereby activating specific plant secondary metabolic pathways, synthesis and accumulation of specific active ingredients. Such as the periwinkle endophytes can induces the vinblastine synthesis and accumulation, production increased by 2-5 times. Symbiosis between the plant and the plant can not be ignored, a plant grown in a particular place, there must be the existence of their mutual symbiotic plants. After a certain period of its growth, the local plant symbiotic changes or does not exist, then the medicine will occurs transition, to the suitable growth place, so pay attention to the symbiotic state impact on medicine quality.

\section{THE CHARACTERISTICS OF THE ORIGINAL ECOLOGICAL PLANTING}

With the increasing amount of herbs, the genuine traditional Chinese medicine increasingly poor, to ensure the clinical use and domestic and international market demand, the study of the ecological environment of genuine traditional Chinese medicine, planting techniques, and create specific production conditions, for the development of high-quality medicine production, develop new sources of drugs are necessary. Chinese medicine in the history is the original ecosystem, the original ecological planting of medicine is simulated wild environment, such as regional, climate, symbiotic, time, etc, irregular watering, fertilizing, conduct field management, etc., to carry out the original ecological planting of traditional Chinese medicine, in order to ensure the quality of traditional Chinese medicine. Some also called bionic medicine planting, ecological planting. The "nature" of Chinese herbal medicines is to adapt to the natural world and product some active substances, and the role of these active substances are the material basis for the majority of Chinese medicine, in order to meet the growing demand for herbal medicines, traditional Chinese medicine of the original ecological planting proposed be imperative.

Original ecological planting is a tradition of philosophy, follow the laws of nature, the analog original ecological characteristics, for example, area, climate, symbiosis, quality and other characteristics, to carry out the original ecological planting of traditional Chinese medicine.TCM original ecological planting is an ecological cropping patterns, it should have the basic characteristics of regional, security and efficiency, etc. The regional refers to the original ecological planting need to local conditions, choose the original production area or similar to the production area, establish of the optimal planting patterns. Security is important features to ensure that clinical use of Chinese herbal medicines, there is awareness of the culture to cultivate of some symbiotic medicinal plants, animals, etc., to avoid contamination of herbs pesticide residues, heavy metals, microbes and other toxic ingredients, thus ensuring the quality of medicines, to ensure medication safety. Efficiency refers to the original ecological planting of 
traditional Chinese medicine is an ecological cropping patterns, are in compliance with the premise of ecological and economic laws, a planting pattern comprehensive planning, its ecological and economic benefits.

\section{CONCLUSIONS}

The genuine traditional Chinese medicine is a product formed by history, it is a phenomenon unique to Chinese traditional medicine, emphasizing the origin and clinical efficacy of herbs, it is a unique evaluation standard quality medicines for ancient. But with the development of society, the environment changes, the mutation of medicine itself, the genuine traditional Chinese medicine of the original production area is constantly changing. The changes of herbs areas is the inevitable result of social development, it can meet the needs of people for genuine medicine in some extent. But at the same time the clinical demand for genuine traditional Chinese medicine increasing, making it excessive pursuit of production, while ignoring its growing conditions for the intrinsic quality of genuine traditional Chinese medicine. And some of the existing GAP cultivation of Chinese herbal medicines, although increased the production of medicines, recognizes the importance of quality, but lost the advantage of a good of traditional Chinese medicine planting, such as the growth of traditional Chinese medicine is need mutually symbiotic with medicinal plants in nature, animals ,it can exist the cure active substance. For medicine we should be based on modern science and technology, and orderly conduct of the original ecological planting of Chinese herbal medicines, promoting the sustainable use of herbal medicines, maintain the ecological balance. Also in the original ecological planting, increase the research of endogenous bacteria and symbiotic plants, it can promote the formation mechanism of genuine traditional Chinese medicine reveals, achieve the large-scale of efficient and highquality cultivation.TCM original ecological planting clear direction for the sustainable development of the genuine traditional Chinese medicine, it making medicines make greater contributions to human health. How will the original ecological planting of Chinese medicine combined with GAP, Which is to ensure the quality and clinical efficacy of medicines, but also to ensure their production, meet the growing demand for herbal medicines, and it has a broad prospects, it should be the focus of future research.

\section{REFERENCES}

[1] Ting He, Ying Gong, Wenya Liu.2014.Characteristics connotation of genuine medicine. Modern Chinese Medicine21(2):58-60.

[2] Shuo Tian, Mingsan Miao.2014.Genuine Chinese Herb and Its Evolution. Chinese Journal of Chinese Medicine 29(4):554-556.

[3] Fei Lang, Jian Li, Wei Zhang. 2013. Brief introduction of geo-authentic herbs. China Journal of Chinese Materia Midica38 (09):1455-1457.

[4] Ke Zhong, Wenquan Wang, Fengyun Jin. 2013. Discussion of Anemarrhena asphodeloides geoherbs historiography. Information on Traditional Chinese Medicine30(01):29-33.

[5] Cheng Peng.2011.The elements and the crisis faced by the formation of authentic ingredients. Pharmacy and Clinics of Chinese Materia Medica2(1):7-10.

[6] Wenjing $\mathrm{Xu}$.2014.The influence factors on the quality of Chinese medicinal materials. Contemporary Medicine Forum12(7):56-57.

[7] Xiajun Zhong, Yuanfeng Tan.2012.Research progress of soil factor influences on the quality of genuine medicinal materials. JOURNAL OF SOUTHERN AGRICULTURE43 (11):1708-1711.

[8] Hong Jiang, Qinzeng Zhang, Lijun Jie. 2014. Acute toxicity studies of Hebei authentic ingredients Aster. Journal Med Pest Control30 (3):288-289.

[9] Xiaohe Xiao, Shanglin Chen, Luqi Huang.2009.Survey of investigations on Daodi Chinese medicinal materials in China since1980s.China Journal of Chinese Materia Midica34(5):519-523.

[10] Bao Liu, Lingling Yang, inghong Wang. 2009. Effects of microorganisms on plant effective components of traditional Chinese medicine formation source. Natural Product Research and Development21:1065-1068, 933.

[11] Wei Wu, Chunji Zhang, Mingsan Miao. 2011. Study on Genuine Traditional Chinese Assessment Mode. CHINA JOURNAL OF CHINESE MEDICINE26 (154):339-341.

[12] Qingsheng Wu, Renbin Zhu, Zhihu Wan. 2002. The Correlation between Effective Components of American Gin-seng and Climatic Factors. Acta Ecologica Siniga22 (5):779-782.

[13] Caixiang Xie, Fengmei Suo, Yingqun Zhou.2011. Quantitative study on ecological suitability of Chinese herbalmedicine based on GIS. China Journal of Chinese Materia Midica36 (3):379-382. 